\title{
"Taylor expansion for derivative securities pricing as a precondition for strategic market decisions"
}

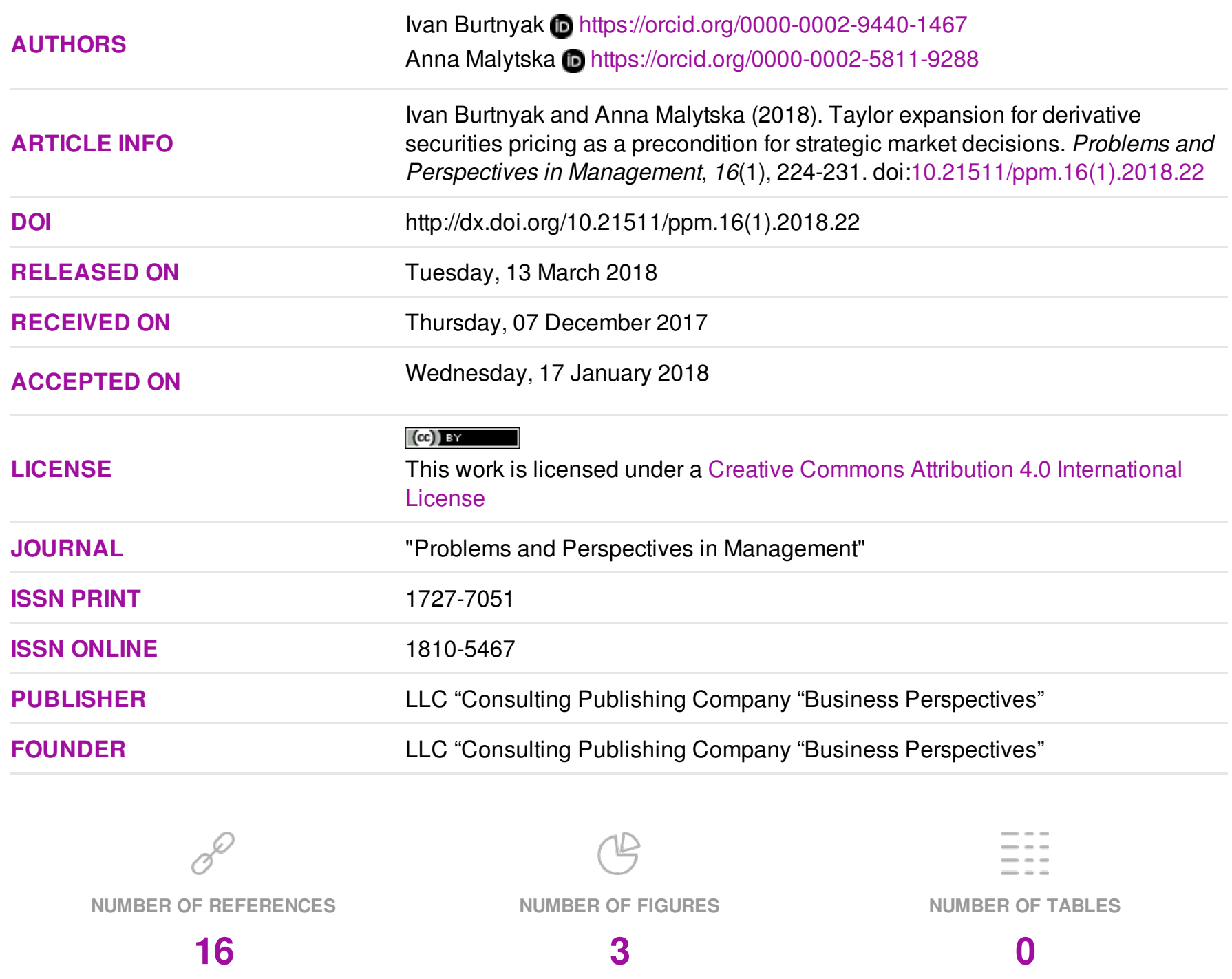

(C) The author(s) 2022. This publication is an open access article. 


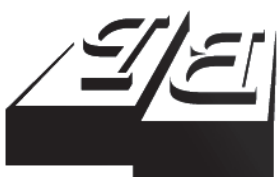

BUSINESS PERSPECTIVES

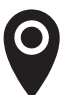

LLC “CPC "Business Perspectives” Hryhorii Skovoroda lane, 10, Sumy, 40022, Ukraine

www.businessperspectives.org

Received on: $7^{\text {th }}$ of December, 2017 Accepted on: $17^{\text {th }}$ of January, 2018

(C) Ivan Burtnyak,

Anna Malytska, 2018

Ivan Burtnyak, Ph.D. (Economics), Associate Professor, Department of Economic Cybernetics, Vasyl Stefanyk Precarpathian National University, Ukraine.

Anna Malytska, Ph.D. (Physics and Mathematics), Associate Professor, Department of Mathematical and Functional Analysis, Vasyl Stefanyk Precarpathian National University, Ukraine.

\section{() (1)}

This is an Open Access article, distributed under the terms of the Creative Commons Attribution 4.0 International license, which permits unrestricted re-use, distribution, and reproduction in any medium, provided the original work is properly cited.

\title{
TAYLOR EXPANSION FOR DERIVATIVE SECURITIES PRICING AS A PRECONDITION FOR STRATEGIC MARKET DECISIONS
}

\begin{abstract}
The strategy of managing the pricing processes, in particular managing the dynamics of the price of the underlying asset and its volatility, the prices of indices, shares, options, the magnitude of financial flows, in the method of calculating the company's rating based on the available quotations of securities, is developed. The article deals with the study of pricing and calculating the volatility of European options with general local-stochastic volatility, applying Taylor series methods for degenerate diffusion processes. The application of this idea requires new approaches caused by degradation difficulties. Price approximation is obtained by solving the Cauchy problem of partial differential equations diffusion with inertia, and the volatility approximation is completely explicit, that is, it does not require special functions. If the payoff of options is a function of only $\mathrm{x}$, then the Taylor series expansion does not depend on $\mathrm{t}$ and an analytical expression of the fundamental solution is considerably simplified. Applied an approach to the pricing of derivative securities on the basis of classical Taylor series expansion, when the stochastic process is described by the diffusion equation with inertia (degenerate parabolic equation). Thus, the approximate value of options can be calculated as effectively as the Black-Scholes pricing of derivative securities. On the basis of the solved problem, it is possible to calculate their turns step-by-step. This enables to predict the dynamics of the pricing of derivatives and to create a strategy of behavior at options according to the passage of the process. For each approximation, price volatility is calculated, which makes it possible to take into account all changes in the market and to calculate possible situations. The step-by-step finding of the change in yield and volatility in the relevant analysis enables us to make informed strategic decisions by traders in the financial markets.
\end{abstract}

Keywords

\section{JEL Classification C41, C32, D49, G17}

\section{INTRODUCTION}

Modeling and forecasting of pricing dynamics by using financial instrument are an important element of investment activity, as they have a high level of financial leverage and fulfillment of obligations is realized in the future. Derivative prices tend to change and predicting their behavior is becoming more and more complicated. Stochastic processes that are described by the equation of diffusion with inertia are widely used in the theory of mass service, in particular in the theory of queues. Often, such processes occur in financial markets with the pricing of European and Asian options. The theory of pricing derivatives and the study of the behavior of volatility for the analysis of profitability are necessary for flexibility in the adoption of managerial strategic decisions by managers. The validity of strategic decisions allows managers to make step-by-step additional investments in order to maintain strategic positions of the company in the stock market. Typically, a high level of volatility gives managers more opportunities to change their decisions in the future. Volatility is important for trad- 
ers when pricing several different series of options with different execution rates and maturities. Since it is stable in the Black-Scholes model (Black \& Scholes, 1973), speculative price changes can occur in these cases, which cannot be verified without the presence of a certain kind of specialized knowledge. This is one example of how volatility pricing options are related to the principles of modeling and risk management in the financial market. Therefore, it is necessary to use models with variable volatility.

The purpose of this article is to introduce a unified approach to pricing and find the implied volatility of European options, which are described by degenerate diffusion processes of diffusion type with inertia, based on classical Taylor series approximation. Therefore, the approximate value of options can be calculated as effectively as the price of Black-Scholes (Black \& Scholes, 1973) for European options.

\section{LITERATURE REVIEW}

Various operations with financial products suitable for selling and buying are carried out using derivative financial instruments (Aboulaich et al., 2013). Securities market participants should have a good understanding of derivative pricing to achieve successful financial results. Derivative securities transactions occupy an important place in the stock market financial activity, since each participant must hedge the risks to obtain extra profit based on stock market speculations (Anderson, 2011). Therefore, the derivatives are one of the major instruments in the securities market. An important task is to study the state and dynamics of the domestic stock market in close interrelation with other countries' stock markets and analyze the volatility of financial instruments to increase the efficiency of investment operations. Nowadays many approaches have been developed to calculate local and stochastic volatility that describe the overall dynamics of underlying price using CEV models (Lindsay \& Brecher, 2012; JDCEV Carr \& Linetsky, 2006), Heston model (Heston, 1993), SABR-model (Ren et al., 2007), but the application of these models requires the use of special functions and numerous integrations of complex functions. Hence, this may lead to miscalculations, but considering the time law (dependency), a considerable number of the models become unstable. Other methods for pricing derivative securities are required to get direct calculations (Forde et al., 2012). The use of Taylor series expansion depends on the model structure, specifically, on function properties, which are used in the model, and model ability to maintain the stability at time change.

The article considers models without default of diffusion process with inertia, with coefficients depending on the variables $(t, x, y)$; for derivatives pricing we use Taylor series expansion for degenerate diffusion processes. In particular, complete correlation matrices are non-degenerate in the works by Mendoza-Arriagaetal (2010), Lorig (2013), and Lorigetal (2013), and in our work they are degenerate, so the application of this idea requires new approaches caused by degradation difficulties.

\section{METHODS}

Consider the market without arbitration, zero interest rate and with no dividends. Without losing generality, these considerations may be extended to deterministic interest rate. Probabilistic space is used with martingale measure $\Xi$, with filtering $\left\{\mathcal{F}_{t}, t \geq 0\right\}$ defining market history. Let asset $S$ represent phenomena such as stocks, price index, a reliable short-term interest rate, i.e. and where $S_{t}=\mathbb{I}_{\{\tau>t\}} \mathrm{e}^{X_{t}}$, and processes $X_{t}$ and $Y_{t}$ are set by the system of equations:

$$
\begin{aligned}
& \mathrm{d} X_{t}=\mu\left(t, X_{t}, Y_{t}\right) d t+ \\
& +\sigma\left(t, X_{t}, Y_{t}\right) d B_{t}, X_{0}=x \in R, \\
& \mathrm{~d} Y_{t}=\alpha\left(t, X_{t}, Y_{t}\right) d t, Y_{0}=y \in R,
\end{aligned}
$$

where $\tau$ is the stopping time

$$
\tau=\inf \left\{t \geq 0: \int_{0}^{t} r\left(s, X_{s}, Y_{s}\right) d s \geq \varepsilon\right\},
$$

with the exponential distribution $\varepsilon$, which does not depend on $X$, the drift function $\mu$ has the form: 
$\mu\left(t, X_{t}, Y_{t}\right)=-\frac{1}{2} \sigma^{2}\left(t, X_{t}, Y_{t}\right)+r\left(t, X_{t}, Y_{t}\right)$

Let $U$ be an on-arbitrary price of the European option, which at time $T$ is a gain $\mathcal{K}\left(S_{T}\right)$ (Jeanblanc et al., 2009) that:

$U_{t}=K+\mathbb{I}_{\{\tau>t\}} \mathbb{E}\left\{\mathrm{e}^{-\int^{\mathrm{T}}{ }^{-}\left(s, X_{s}, Y_{s}\right) d s}\left(k\left(X_{t}\right)-\mathrm{K}\right) \mid X_{t}, Y_{t}\right\}$,

$t<T$.

$K=\mathcal{K}(0), k(x)=\mathcal{K}\left(e^{x}\right)$.

To calculate the price of the European option, we need to find the mathematical expectation from

$$
\begin{aligned}
& e^{-\int_{\mathrm{t}}^{\mathrm{T}} r\left(s, X_{s}, Y_{s}\right) d s}\left(k\left(X_{t}\right)-\mathrm{K}\right) \text { in particular } \\
& w(t, x, y)= \\
& =\mathbb{E}\left\{e^{-\int_{\mathrm{t}}^{\mathrm{T} r\left(s, X_{s}, Y_{s}\right) d s}}\left(k\left(X_{t}\right)-\mathrm{K}\right) \mid X_{t}=x, Y_{t}=y\right\} .
\end{aligned}
$$

The function $w(t, x, y)$ satisfies the Kolmogorov equation of diffusion with inertia

$$
\begin{aligned}
& \left(\partial_{t}-x \partial_{y}+P\right) w= \\
& =0,\left.w(T, x, y)\right|_{t=T}=k(x, y),
\end{aligned}
$$

where operator $P$ has the form:

$$
P=a(t, x, y)\left(\partial_{x}^{2}-\partial_{x}\right)+r(t, x, y)\left(\partial_{x}-1\right),
$$

where $a(t, x, y)$ is equal to:

$$
a(t, x, y):=\frac{1}{2} \sigma^{2}(t, x, y) \text {. }
$$

For deterministic interest rates, these formulas are easy to propagate as they look similar. An approximate solution to the Cauchy problem for diffusion equation with inertia (3) is obtained by adapting the ideas of Lorig et al. (2013) and Pascucci (2011) which are applied to non-degenerate diffusion processes. We consider degenerate diffusion processes with inertia on which singular integro-differential pricing operators of Levy type are applied. We presented a unified approach to pricing and esti- mation of implicit volatility using Taylor series expansion. Consider that $a$ and $r$ are infinitely differentiable functions of variables $(x, y)$, continuous on $t$ and bounded $\forall(t, x, y) \in[0, T] \cdot R^{2}$.

Let $(\bar{x}, \bar{y}) \in R^{2}$ be a fixed point then for any analytical function $h=h(t, x, y)$ it is necessary to write down Taylor series expansion:

$$
\begin{aligned}
& k(t, x, y)=\sum_{n=0}^{\infty} \sum_{l=0}^{n} k_{n-l, l}(t)(x-\bar{x})^{n-l}(y-\bar{y})^{l}, \\
& k_{n-l, l}(t):=\frac{1}{(n-l) ! l !} \partial_{x}^{n-l} \partial_{y}^{l} k(t, \bar{x}, \bar{y}) .
\end{aligned}
$$

Applying the above considerations to the coefficients $a$ and $r$, the operator received $P$ in (4) has the form:

$P=\sum_{n=0}^{\infty} P_{n}, P_{n}:=\sum_{l=0}^{n}(x-\bar{x})^{n-l}(y-\bar{y})^{l} P_{n-l, l}$,

where $\left\{P_{n-l, l}\right\}$ are differential operators of the

$$
P_{n-l, l}:=a_{n-l, l}(t)\left(\partial_{x}^{2}-\partial_{x}\right)+r_{n-l, l}(t)\left(\partial_{x}-1\right) .
$$

Operator $P$ of a parabolic type $x$ and has a degenerate parabolicity on $y$ (since there is no second order derivative on $y$ ), which usually operates in financial spaces, that is, in the financial markets. Based on Taylor series expansion for $P$, the pricing function $w$ has the following form (Corielli et al., 2010):

$$
w=\sum_{n=0}^{\infty} w_{n} .
$$

Substituting (5) and (6) into (3), we obtain the Cauchy problems for diffusion equations with inertia.

$\left(\partial_{t}-x \partial_{y}+P_{0}\right) w_{0}=0, w_{0}(T, x, y)=k(x, y)$,

$\left(\partial_{t}-x \partial_{y}+P_{0}\right) w_{n}=-\sum_{k=1}^{n} P_{k} w_{n-k}$,

$w_{n}(T, x, y)=0$,

A fundamental solution was constructed on the homogeneous Cauchy problem (7) and it uses the properties of the fundamental solution (7) to find $w_{n} \forall n$ as a solution of the problem (8). The properties of the distribution function for the diffusion 
equation with inertia expressed by the classical Chapman-Kolmogorov-Planck equations and the Duhamel's principle are applied to partial derivatives equations.

Let's consider the Cauchy problem (7). Operator $P_{0}$ is a degenerate parabolic diffusion operator with inertia, or a Kolmogorov operator with timedependent coefficient $t$. Thus, the solution $w_{0}$ takes the form

$$
\begin{aligned}
& w_{0}(t, x, y)=e^{\int^{\mathrm{T}} r_{0}(s) d s} \times \\
& \times \int_{R^{2}} \mathcal{E}_{0}(t, x, y ; T, \xi, \eta) k(\xi, \eta) d \xi d \eta,
\end{aligned}
$$

where $\mathcal{E}_{0}(t, x, y ; T, \xi, \eta)$ is a two-dimensional Gaussian density of diffusion process with inertia.

To find $\mathcal{E}_{0}(t, x, y ; T, \xi, \eta)$, sow solve the Cauchy problem for the diffusion equation with inertia and variable coefficients depending on $t$

$$
\begin{aligned}
& \partial_{t} w-x \partial_{y} w+a_{0}(t)\left(\partial_{x}^{2} w-\partial_{x} w\right)+ \\
& +r_{0}(t)\left(\partial_{x}-1\right) w=0 . \\
& \left.w(t, x, y)\right|_{t=T}=k(x, y) .
\end{aligned}
$$

Make a Fourier transform to solve the Cauchy problem (10), (11) assuming that $w, w_{t}, w_{x}$, $w_{x^{2}}, w_{y}$ are completely integrable on $(x, y)$. We sum up the system then the problems (10) and (11) were reduced to the Cauchy problem for a linear differential equation with partial derivatives of the first order (Gatheral et al., 2012). Having solved this problem and taking the inverse Fourier transform from its solution, we obtain the solutions (10), (11):

$w(t, x, y)=\int_{R^{2}} k\left(x^{\prime}, y^{\prime}\right) e^{\int^{T} r_{0}(\beta) d \beta} \times$

$\times \mathcal{E}_{0}\left(t, x, y ; T, x^{\prime}, y^{\prime}\right) d x^{\prime} d y^{\prime}$

where $\mathcal{E}_{0}(t, x, y ; T, \xi, \eta)$ is the density of the two-dimensional diffusion process with inertia and with coefficients dependent on a time variable.

$$
\begin{aligned}
& \mathcal{E}_{0}\left(t, x, y ; T, x^{\prime}, y^{\prime}\right)=\left(4 \pi c^{*}\right)^{-1}\left(\int_{t}^{T} a_{0}(\beta) d \beta\right)^{-\frac{1}{2}} \times \\
& \times \exp \left\{-\left(4 \int_{t}^{T} a_{0}(\beta) d \beta\right)^{-1} \times\right. \\
& \times\left(x^{\prime}-x-\int_{t}^{T}\left(a_{0}(\beta)-r_{0}(\beta)\right) d \beta\right)^{2}- \\
& -\left(2 c^{*}\right)^{-2}\left[y^{\prime}-y+x(T-t)-\right. \\
& -\left(x^{\prime}-x-\int_{t}^{T}\left(a_{0}(\beta)-r_{0}(\beta)\right) d \beta\right) \times \\
& \times \int_{t}^{T}(\beta-T) a_{0}(\beta) d \beta\left(\int_{t}^{T} a_{0}(\beta) d \beta\right)^{-1}- \\
& \left.\left.-\int_{t}^{T}(\beta-T)\left(a_{0}(\beta)-r_{0}(\beta)\right) d \beta\right]^{2}\right\}, \\
& 0 \leq t<T,(x, y) \in R^{2},\left(x^{\prime}, y^{\prime}\right) \in R^{2},
\end{aligned}
$$

where $(t, x, y)$ is the initial point, $\left(T, x^{\prime}, y^{\prime}\right)$ is the end point (current).

$$
\begin{aligned}
& c^{* 2}=\int_{t}^{T}(\beta-T)^{2} a_{0}(\beta) d \beta- \\
& -\left(\int_{t}^{T}(\beta-T) a_{0}(\beta) d \beta\right)^{2}\left(\int_{t}^{T} a_{0}(\beta) d \beta\right)^{-1} .
\end{aligned}
$$

First you need to solve the Cauchy problem (8) with $n=1$, pay off $=\sigma(X, Y), w(t, x, y)=\mathcal{E}_{0}(t, x, y ; T, X, Y)$.

$w_{1}(t, x, y) e^{\int^{T} r_{0}(s) d s}=\int_{\mathrm{t}}^{\mathrm{T}} d s \int_{R^{2}} d \xi d \eta \mathcal{E}_{0}(t, x, y ; s, \xi, \eta) \times$ $\times P_{1} \mathcal{E}_{0}(s, \xi, \eta ; T, X, Y)=$ $=\int_{\mathrm{t}}^{\mathrm{T}} d s B_{1}^{(x, y)}(t, s) \mathcal{E}_{0}(s, \xi, \eta ; T, X, Y)$.

Multiplying both sides by $e^{\int^{T} r_{0}(s) d s}$ and using (9) we have

$$
w_{1}(t, x, y)=A_{1} w_{0}(t, x, y), A_{1}:=\int_{\mathrm{t}}^{\mathrm{T}} d s B_{1}(t, s) .
$$

Using method of mathematical induction and 
taking into consideration properties of the fundamental solution we received

$$
\begin{aligned}
& w_{n}(t, x, y)=A_{n} w_{0}(t, x, y): \\
& A_{n}:=\sum_{k=1}^{n} \int_{t}^{T} d s_{1} \ldots \int_{s_{n-1}}^{T} d s_{n} \sum_{i \in I_{n, k}} B_{i_{1}}\left(t, s_{1}\right)\left(B\left(t, s_{2}\right)+\right. \\
& +B\left(s_{1}, s_{2}, 0\right)+\left(1+s_{2}-s_{1}\right) B\left(t, s_{1}, 0\right)- \\
& \left.-B\left(t, s_{2}, 0\right)\right)_{i_{2}} \ldots\left(B\left(t, s_{n}\right)+B\left(s_{n-1}, s_{n}, 0\right)+\right. \\
& \left.+\left(1+s_{k-1}-s_{k}\right) B\left(t, s_{k-1}, 0\right)-B\left(t, s_{k}, 0\right)\right)_{i_{k}} \\
& I_{n, k}=\left\{i=\left(i_{1}, \ldots i_{k}\right) \in N^{k} \mid i_{1}+\ldots+i_{k}=n\right\} \\
& 1 \leq k \leq n, \\
& B_{i_{k}}(t, s):=B_{i_{k}}(t, s, x, y, \bar{x}, \bar{y}) \\
& B_{i_{k}}(t, s)_{\mid(x, y)=(\bar{x}, \bar{y})}=B(t, s, 0) \\
& B_{n}(t, s):=\sum_{k=1}^{n} \mathcal{M}_{n-k, k}(t, s) P_{n-k, k}(s) \\
& \mathcal{M}_{k, l}=\left(\mathcal{M}_{1}(t, s)\right)^{k}\left(\mathcal{M}_{2}(t, s)\right)^{l}
\end{aligned}
$$

where

$$
\begin{aligned}
& M_{1}(t, s):=\left(x-X+\int_{t}^{s}\left(a_{0}(\beta)-r_{0}(\beta)\right) d \beta+\right. \\
& +2 \int_{t}^{s} a_{0}(\beta) d \beta \partial_{x}+2\left(\int_{t}^{s}(\beta-s) a_{0}(\beta) d \beta+\right. \\
& \left.\left.+(s-t) \int_{t}^{s} a_{0}(\beta) d \beta\right) \partial_{y}\right)^{n}
\end{aligned}
$$

The results of the asymptotic approximation are proved in Pagliarani et al. (2013) and Lorig et al. (2013). These formulas are considerably simplified when the coefficients do not depend on time.

\section{RESULTS}

The article considers the degenerate diffusion process that describes price dynamics and implicit volatility, depending on time and $(t, x, y)$, indexes of base assets, stocks, options of financial flows in the method of calculating the company's rating based on the available quotations of securities. The research was conducted on the basis of Taylor series. The density distribution of the probabilities of passing this process is constructed. With density distribution, one can find $\mathcal{E}_{0}$ and the price $w(t, x, y)$ at any given time. The method of successive finding of the price is developed when the coefficients depend on $(t, x, y)$

- Using approximate approximations considering the Black-Scholes price function as an initial approximation, we obtain explicit formulas for finding the initial approximations of implicit volatility. We note that they in the form of the record match the formulas (Lorig et al., 2013), but in this case they are more complicated by the degeneration of the equation and the formula for the approximation of the price.

The obtained implications of implicit volatility and consistent price approximations make it possible to analyze the process of passage in the financial market. Let as make corrections and concrete steps to improve the situation for optimizing financial strategies.

$$
\begin{aligned}
& M_{2}(t, s):=\left(y-Y-\int_{t}^{s}(\beta-s) a_{0}(\beta) d \beta\left(\int_{t}^{s} a_{0}(\beta) d \beta\right)^{-1} \int_{t}^{s}\left(a_{0}(\beta)-r_{0}(\beta)\right) d \beta+\right. \\
& +\int_{t}^{s}(\beta-s)\left(a_{0}(\beta)-r_{0}(\beta)\right) d \beta+2 \int_{t}^{s}(\beta-s) a_{0}(\beta) d \beta \partial_{x}+2 c^{* 2} \partial_{y}+ \\
& \left.+\left(2 \int_{t}^{s}(\beta-s) a_{0}(\beta) d \beta\left(\int_{t}^{s} a_{0}(\beta) d \beta\right)^{-1}\right)\left(\int_{t}^{s}(\beta-s) a_{0}(\beta) d \beta+(s-t) \int_{t}^{s} a_{0}(\beta) d \beta\right) \partial_{y} 2 c^{* 2}\right)^{n} .
\end{aligned}
$$




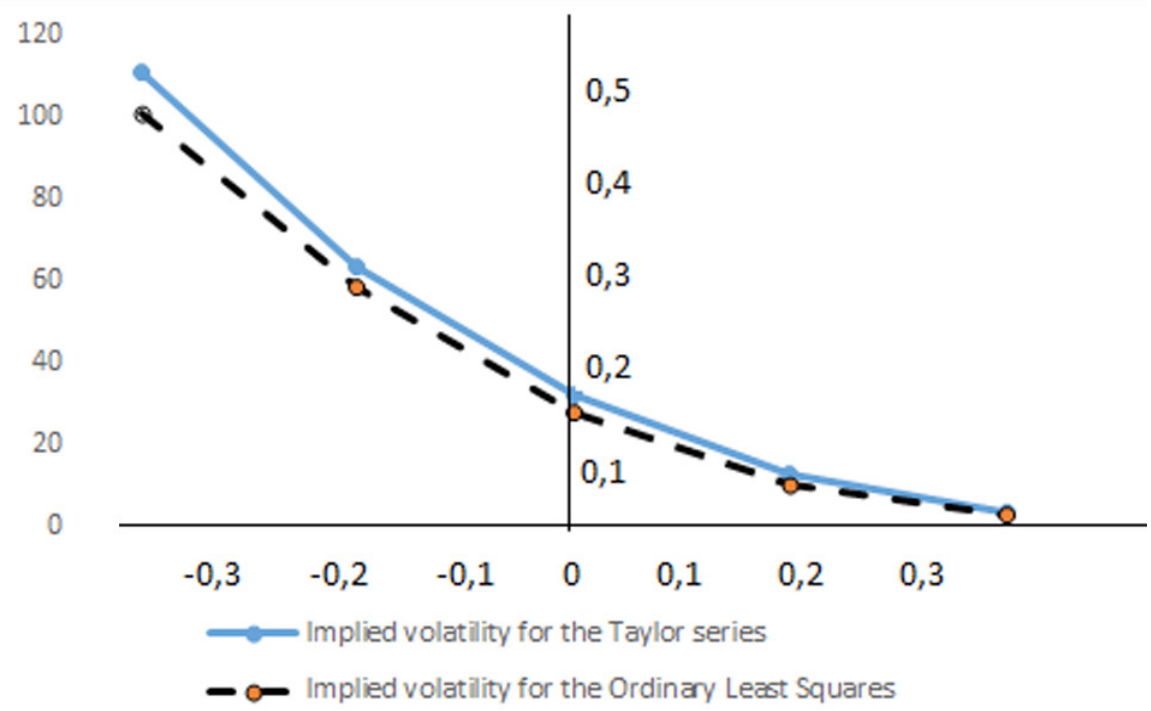

Figure 1. The implied volatility obtained by the Ordinary Least Squares and our second order approximation by the Taylor series for the degenerate Heston model $e^{y}=0,249^{2}, T=0.125, t=0$

A Taylor series expansion method for the lows managers to predict the process of forming a Kolmogorov equation (degenerate Heston model) portfolio, financial flows, and changes in pricing. is used

$$
\partial_{t} w-x \partial_{y} w+\frac{e^{y}}{2}\left(\partial_{x}^{2} w-\partial_{x} w\right)=0
$$

with the initial function, which is the Black-Scholes value-dependent volatility based on S\&P500 options data in the time interval from November 13 to December 29, 2017. The exact values of changes in volatility and profitability are found, which al-

In the example of the degenerate Heston model, which describes the dynamics of pricing and the development of implicit volatility, the initial approximation of the Black-Scholes price function made volatility and yield calculations based on the second Taylor approximation and the Ordinary Least Squares. The results obtained are almost identical, indicating a high accuracy of approximation.

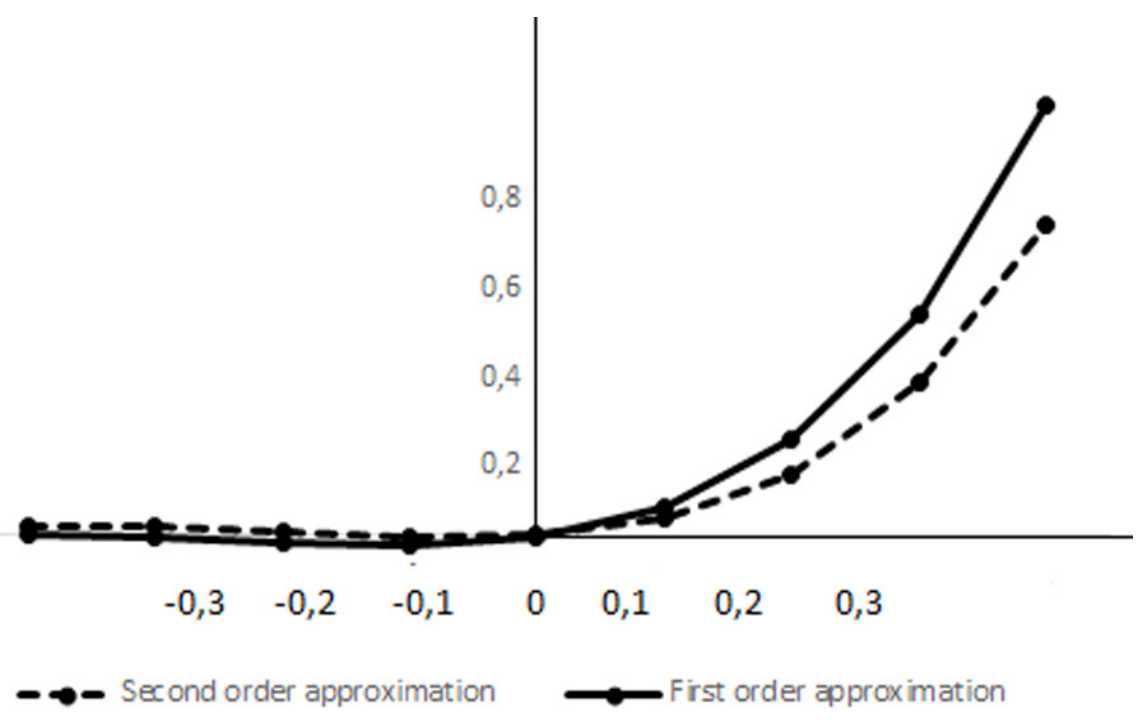

Figure 2. The yield curve of first and second order approximation, the Taylor series for the degenerate Heston model $e^{y}=0,249^{2}, T=0.125, t=0$ 
Knowledge of the approximate price and implied volatility at each step at a fixed time gives an opportunity to develop a strategy for managing the dynamics of derivative prices in financial markets and to avoid speculative changes in pricing.

The density of distribution for degenerate diffusion processes is constructed when the coefficients depend only on time (7).
The method of constructing the density of distribution in the case of coefficients dependence on time and spatial variables is developed (3), when the correlation matrix is not strictly definite positive.

Knowing the density of distribution, one can always find the price by the formula (13) where the function of $\mathcal{E}_{0}$ is fulfilled by the density of distribution of probabilities of the investigated economic process.

\section{CONCLUSION}

This paper expands the methodology of approximate pricing for a wide range of derivative assets. Price approximation is obtained by solving the Cauchy problem for differential equations in partial derivatives of diffusions with inertia. If the payoff of options is a function of only $\mathrm{x}$, then the Taylor series expansion does not depend on $t$ and analytical expression of the fundamental solution of the distribution density of the stochastic process is considerably simplified. We have applied an approach to the pricing of European options on the basis of classical Taylor series expansion, when the stochastic process is described by the diffusion equation with inertia (degenerate parabolic equation). For a degenerate parabolic equation, the approximate price of options is fairly simple since it uses only estimates of derivatives of the distribution density of diffusion with inertia obtained by explicit formulas for European options price approximations.

The approach to the pricing of derivatives of securities and the finding of implicit volatility based on the classical approximation of the Taylor series when the stochastic process is described by the equation of diffusion with inertia (degenerate parabolic equation) is introduced. For a degenerate parabolic equation, finding the approximate price of options is fairly simple since it uses only estimates of derivatives of the distribution density of diffusion with inertia.

The step-by-step finding of the change in yield and volatility in the relevant analysis enables us to make informed strategic decisions by traders in financial markets.

\section{REFERENCES}

1. Aboulaich, R., Baghery, F., \& Jrai A (2013). Option Pricing for a Stochastic Volatility Jump-Diffusion Model. International Journal of Mathematics and Statistics, 13(1), 1-19.

2. Andersen, L. (2011). Option pricing with quadratic volatility: a revisit. Finance and Stochastic, 15(2), 191-219.

3. Black, F., \& Scholes, M. (1973). The Pricing of Options and Other Corporate Liabilities. Journal of Political Economy, 81(3), 637-654. Retrieved from https://www. cs.princeton.edu/courses/archive/ fall09/cos323/papers/black_scholes73.pdf
4. Carr, P., \& Linetsky, V. (2006). A jump to default extended CEV model: An application of Bessel processes. Finance and Stochastics, 10(3), 303-330.

5. Corielli, F., Foschi, P., \& Pascucci, A. (2010). Parametrix approximation of diffusion transition densities. SIAM Journal on Financial Mathematics, 1, 833-867.

6. Forde, M., Jacquier, A., \& Lee, R. (2012). The small-time smile and term structure of implied volatility under the Heston model. SIAM Journal on Financial Mathematics, 3(1), 690-708.
7. Gatheral, J., Hsu, E. P., Laurence, P., Ouyang, C., \& Wang, T.H. (2012). Asymptotics of implied volatility in local volatility models. Mathematical Finance, 22(4), 591-620.

8. Heston, S. (1993). A Closed-Form Solution for Options with Stochastic Volatility with Applications to Bond and Currency Options. Review of Financial Studies, 6(2), 327-343. Retrieved from http:// citeseerx.ist.psu.edu/viewdoc/dow nload? $\mathrm{doi}=10.1 \cdot 1 \cdot 139.3204 \& \mathrm{rep}=\mathrm{r}$ ep1\&type $=$ pdf

9. Jeanblanc, M., Yor, M., \& Chesney, M. (2009). Mathemati- 
cal methods for financial markets. Springer Verlag.

10. Lindsay, A. E., \& Brecher, D. R. (2012). Simulation of the CEV Process and the Local Martingale Property. Mathematics and Computers in Simulation, 82, 868-878.

11. Lorig, M. (2013). The exact smile of certain local volatility models. Quantitative Finance, 13(6), 897-905.
12. Lorig, M., Pagliarani, S., \& Pascucci, A. (2013). A Taylor series approach to pricing and implied vol for LSV models. Working Paper available at ArXiv. Retrieved from http://arxiv.org/ abs/1308.5019

13. Mendoza-Arriaga, R., Carr, P., \& Linetsky, V. (2010). Time-changed Markov processes in unified creditequity modeling. Mathematical Finance, 20, 527-569.
14. Pagliarani, S., Pascucci A., \& Riga, C. (2013). Adjoint expansions in local Levy models. SIAM Journal Financial Mathematics, 4, 265-296.

15. Pascucci, A. (2011). PDE and martingale methods in option pricing. Bocconi \& Springer Series. New York: Springer-Verlag.

16. Ren, Y, Madan, D., \& Qian, M. Q. (2007). Calibrating and pricing with embedded local volatility models. Risk, 138-143. 